\title{
Acute pericarditis due to perforation of a benign mediastinal teratodermoid into the pericardial sac
}

\author{
IM PATERSON, JS COCKBURN \\ From the Department of Cardiothoracic Surgery, Aberdeen Royal Infirmary, Aberdeen
}

Rupture into the pericardium is a very rare complication of anterior mediastinal teratodermoid. There have been only four recorded cases, which either have been characterised by cardiac tamponade or have been an incidental finding at operation or necropsy. This report concerns a patient who presented with acute pericarditis and is considered to be unique.

\section{Case report}

A 32-year-old woman was admitted to hospital with a threehour history of anterior chest pain. This was sudden in onset, radiated to the neck and arm, and was associated with nausea and vomiting. Before this episode, she had been entirely well.

On admission no abnormality was found during the physical examination. The white cell count was $15 \cdot 0 \times 10^{9} / 1$ with a normal differential count and the erythrocyte sedimentation rate was $22 \mathrm{~mm}$ in one hour (Westergren). The initial electrocardiogram and cardiac enzyme levels were normal but the following day there was widespread ST elevation and the aspartate aminotransferase activity was raised at $360 \mathrm{U} / \mathrm{l}$. An anteroposterior chest radiograph taken shortly after admission showed segmental collapse at the left base but was otherwise considered normal. A presumptive diagnosis of acute pericarditis was made and an echocardiogram was performed. This showed $1.5 \mathrm{~cm}$ of pericardial fluid but no evidence of cardiac tamponade.

A posteroanterior chest radiograph (fig) taken two days after admission showed a small right pleural effusion; a left hilar mass, which on the lateral view was seen to occupy the anterior mediastinum; and a possible right hilar mass. Tomography of this area showed a lobulated mass affecting the left hilum and extending forwards to occupy most of the area between the mediastinum and the anterior chest wall. The appearances were thought to be those of enlarged lymph nodes and in view of the patient's age lymphoma was considered the most likely diagnosis.

Bronchoscopy and mediastinoscopy were performed in an attempt to obtain a tissue diagnosis but the results of both were normal. A left mediastinotomy was therefore carried out by excison of the second costal cartilage. This showed a large cystic mass in the anterior mediastinum, which was closely adherent to the pericardium and mediastinal pleura. When the cyst was opened pultaceous material containing hair was found; digital exploration of the cavity showed

Address for reprint requests: Mr IM Paterson, 85 Drakies Avenue, Inverness, IV2 3SE.

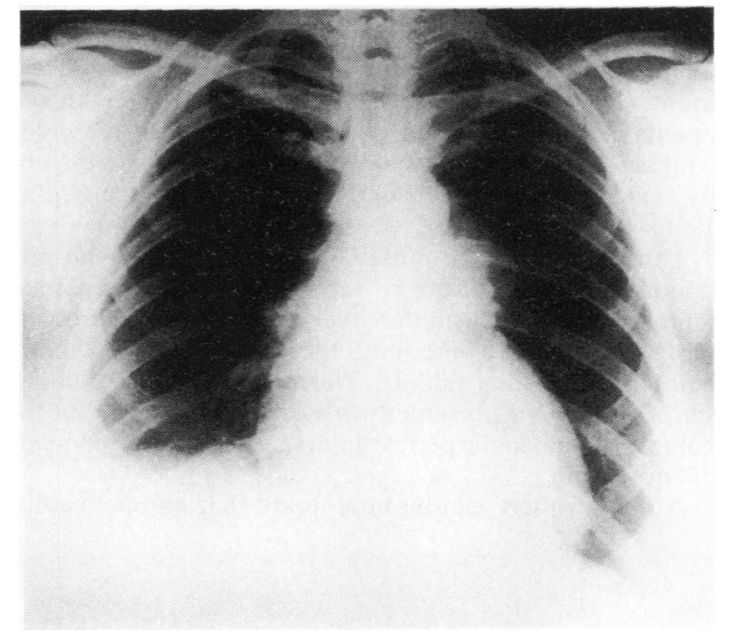

Posteroanterior chest radiograph showing left hilar mass.

extension into the right side of the mediastinum. The skin incision was therefore extended across the midline and the sternum transected to allow removal. It was impossible to separate the cyst from the pericardium, so a large pericardial window was made and the cyst excised in toto.

When the pericardium was opened both serous surfaces were noted to be grossly inflamed with patches of adherent fibrin. The pericardial sac contained hair with pultaceous material similar to that found in the cyst cavity. Although it was obvious that the cyst had ruptured into the pericardial cavity before operation, the point of communication was not found. The histological features were those of a benign mediastinal teratodermoid and the cyst contents were sterile on culture. The patient has remained in good health since the operation.

\section{Discussion}

Perforation into an adjacent structure is an unusual but well recognised complication of anterior mediastinal teratodermoids. The bronchus is the most commonly affected site but rupture into the pleura, aorta, superior vena cava, and pericardium have also been described. 'Marsten et $a l^{2}$ estimated that rupture into adjacent cardiovascular structures by benign teratodermoids occurred in less than $1 \%$ of cases. 
The first recorded case of rupture into the pericardium was described by Cordes in $1859 .{ }^{3}$ The patient had a large pericardial effusion with signs of tamponade and died seven weeks after admission when necropsy showed a mediastinal teratodermoid communicating with the pericardial cavity. More than 50 years later a second report concerned a patient with a large teratodermoid which had perforated into the pleural cavity, causing an empyema. ${ }^{4}$ The intrapericardial rupture was found as an associated incidental finding at necropsy.

The first case to be treated successfully by surgical removal of a perforated cyst was reported by Aktan in $1961 .^{5}$ This patient presented with a five-month history of right-sided chest pain and productive cough and was found at operation to have a teratodermoid compressing the middle-lobe bronchus, causing lobar collapse. The cyst was found to have ruptured into the pericardium when this was opened to allow excision. Another case which presented with cardiac tamponade but which was treated successfully by removal of the cyst was described by Marsten et al in 1966. ${ }^{2}$

Thus in two of the previously recorded cases the predominant feature after rupture has been cardiac tamponade, whereas in the other two the primary problem has been related to the lung. the intrapericardial rupture being an incidental finding. This case is therefore unique since the patient presented with acute pericarditis but the volume of fluid in the pericardium was small and tamponade did not occur.

Although there can be little doubt that intrapericardial rupture has occurred in all the cases described, only Cordes was able to identify the point of communication. ${ }^{3}$ Presumably in other cases the site of rupture was small and sealed spontaneously.

Despite the rarity of the condition, the diagnosis of intra- $\mathbb{D}$ pericardial rupture of a teratodermoid should be considered if there are cardiovascular symptoms and signs with an anterior mediastinal mass on the chest radiograph. The $\vec{\circ}$ accepted treatment, as in uncomplicated cases of teratodermoid. is surgical excision. This can be performed after $\vec{\omega}$ rupture by removing a pericardial window with the teratodermoid. Such a procedure has led to a successful outcome in two previously recorded cases and in the present case.

\section{References}

' Rusby NL. Dermoid cysts and teratomata of the mediastinum: a review. J Thorac Surg 1944;13: 을 169-222.

2 Marsten JL, Cooper AG, Ankeney JL. Acute cardiac tamponade due to perforation of a benign mediastinal teratoma into the pericardial sac: review of cardiovascular manifestations of mediastinal teratomas. Thorac Cardiovasc Surg 1966;51:700-7.

${ }^{3}$ Cordes V. Dermocystoid im mediastinum anticum. Virchows Arch Path Anat 1859:16:29)-306.

+ Masson P. Teratome du mediastin. Bull Soc Anat Paris 1913;77:100-1.

${ }^{5}$ Aktan K. Perikard'a perfore kist dermoid. Turk Tip Cemiy Mecmuasi 1961;27:508-12. 